\title{
Response to Modified open-door laminoplasty for the surgical treatment of cervical spondylotic myelopathy in elderly patients. Biomechanical concerns
}

\author{
Stefan Alexander König
}

Received: 15 July 2014 / Accepted: 17 July 2014 / Published online: 30 July 2014

(C) Springer-Verlag Wien 2014

Dear Editor,

The authors of the article Modified open-door laminoplasty for the surgical treatment of cervical spondylotic myelopathy in elderly patients are aware of the fact that their technique partially compromises the spinal biomechanics. Nevertheless, we do think that the modified laminoplasty has several benefits compared to laminectomy. The refixed laminae do not only provide better protection of the spinal cord than in laminectomy cases, but also serve as an insertion for the posterior neck muscles after surgery. Thus, the posterior tension band might be partially reestablished during the healing process. Therefore, it is desirable to conduct long-term outcome studies that compare the modified technique with conventional laminoplasty and laminectomy.

Sincerely,

Dr. S. Alexander König

Conflict of Interest The authors certify that there is no actual or potential conflict of interest in relation to this article.

\footnotetext{
S. A. König $(\bowtie)$

Neurochirurgische Klinik, Klinikum Karlsruhe, Moltkestr. 90,

76133 Karlsruhe, Germany

e-mail: koenig_de@web.de
} 